\title{
Time Asymmetric Quantum Mechanics
}

\author{
Arno R. BOHM ${ }^{\dagger}$, Manuel GADELLA $\ddagger$ and Piotr KIELANOWSKI ${ }^{\S}$ \\ $\dagger$ Department of Physics, University of Texas at Austin, Austin, TX 78712, USA \\ E-mail: bohm@physics.utexas.edu \\ ¥ Departamento de FTAO, Universidad de Valladolid, 47071 Valladolid, Spain \\ E-mail: manuelgadella1@gmail.com \\ $\S$ Cinvestav, Dept Fis, Mexico City 07000, DF Mexico \\ E-mail:kiel@physics.utexas.edu
}

Received January 30, 2011, in final form August 22, 2011; Published online September 03, 2011 http://dx.doi.org/10.3842/SIGMA.2011.086

\begin{abstract}
The meaning of time asymmetry in quantum physics is discussed. On the basis of a mathematical theorem, the Stone-von Neumann theorem, the solutions of the dynamical equations, the Schrödinger equation (1) for states or the Heisenberg equation (6a) for observables are given by a unitary group. Dirac kets require the concept of a RHS (rigged Hilbert space) of Schwartz functions; for this kind of RHS a mathematical theorem also leads to time symmetric group evolution. Scattering theory suggests to distinguish mathematically between states (defined by a preparation apparatus) and observables (defined by a registration apparatus (detector)). If one requires that scattering resonances of width $\Gamma$ and exponentially decaying states of lifetime $\tau=\frac{\hbar}{\Gamma}$ should be the same physical entities (for which there is sufficient evidence) one is led to a pair of RHS's of Hardy functions and connected with it, to a semigroup time evolution $t_{0} \leq t<\infty$, with the puzzling result that there is a quantum mechanical beginning of time, just like the big bang time for the universe, when it was a quantum system. The decay of quasi-stable particles is used to illustrate this quantum mechanical time asymmetry. From the analysis of these processes, we show that the properties of rigged Hilbert spaces of Hardy functions are suitable for a formulation of time asymmetry in quantum mechanics.
\end{abstract}

Key words: resonances; arrow of time; Hardy spaces

2010 Mathematics Subject Classification: 81Q65

\section{Introduction}

In this article ${ }^{1}$, we describe the notion of time asymmetry (TA) in quantum mechanics, give a brief account of its foundations and formulation and present its relation to the resonance phenomenon.

Time symmetry in quantum mechanics means that the time evolution of states and observables is governed by the unitary group. If we assume that the Hamiltonian $H$ does not depend explicitly on time, then this unitary group is provided by the Stone theorem as $U(t)=e^{-i t H / \hbar}$ with $-\infty<t<\infty$. This situation can be derived from the Hilbert space axiom, which in particular leads to a unitary group of bounded operators: as a consequence of this Hilbert space "boundary condition" for the Schrödinger equation one obtains the unitary group solution for the state.

\footnotetext{
*This paper is a contribution to the Proceedings of the Workshop "Supersymmetric Quantum Mechanics and Spectral Design" (July 18-30, 2010, Benasque, Spain). The full collection is available at http://www.emis.de/journals/SIGMA/SUSYQM2010.html

${ }^{1}$ This manuscript is based on lectures given by A.R. Bohm at the Centro de Ciencias de Benasque, Spain.
} 
If in $U(t)$ the values of $t$ extend from $0 \leq t<\infty$, the evolution is time asymmetric. In this case, time evolution for pure states is not governed by a group. We shall show that in place of the unitary group one has two semigroups. One semigroup with $t \leq 0$ is called the past semigroup, the other, with $t \geq 0$, is called the future semigroup. The physical nature of these semigroups will be discussed in the present paper.

Time evolution governed by a semigroup is not possible in a formulation of quantum mechanics in Hilbert space. The Stone theorem for the solution of the Schrödinger equation and similar for the solution of the Heisenberg equation automatically leads to groups of time evolution in Hilbert space, if the Hamiltonian is self adjoint. Therefore, we have to drop this "Hilbert space boundary condition" and replace it by another type of boundary condition ${ }^{2}$.

A new boundary condition for the dynamical equations is obtained by replacing the Hilbert space by other spaces. This will be the pair of Hardy spaces, $\mathcal{H}_{+}^{2}$ for the Heisenberg equation of the observables and $\mathcal{H}_{-}^{2}$ for the Schrödinger equation. This is a pair of "time asymmetric boundary conditions". Taking into account that we need to extend the Hilbert space into a rigged Hilbert space to implement this time asymmetry, we may call the new boundary condition the "rigged Hilbert space boundary condition". We intend to clarify these notions in the present paper.

The solutions of the Schrödinger equation under the Hilbert space boundary condition

$$
i \hbar \frac{d \phi(t)}{d t}=H \phi(t)
$$

are given by the unitary group

$$
\phi(t)=e^{-i H t / \hbar} \phi(0), \quad \text { for all } t: \quad-\infty<t<+\infty .
$$

This follows from the Stone-von Neumann theorem for the Schrödinger equation (1). Thus in absence of an interaction with an external reservoir no TA is allowed under Hilbert space boundary conditions, i.e., the "Hilbert space boundary condition" does not allow TA.

TA in quantum physics manifests itself in processes like the decay of a quasi-stationary state. TA in these processes is suggested by the fact that the creation or formation of a quasistationary state is not the time reversal of its decay. Formation requires the realization of some initial conditions [1], while the decay is usually spontaneous. Thus, decay can be viewed as an independent process. This process is time asymmetric as we shall discuss later. Both processes (formation as well as decay) are time reversal invariant (except for minute part in $K^{0}$ decay). Resonances are usually produced by a Hamiltonian pair $\left\{H_{0}, H=H_{0}+V\right\}$ where both the "free" Hamiltonian $H_{0}$ and the interaction Hamiltonian $H$ are time reversal invariant, i.e., $\left[A_{T}, H_{0}\right]=0=\left[A_{T}, H\right]$, where $A_{T}$ is the time reversal operator. This means that TA is not to be mistaken for time reversal non-invariance.

The quasi-stationary state undergoes a decay process for which time evolution is defined for $t \geq 0$ only. The quasi-stationary state can be described by a state vector, the Gamow vector which is not normalizable, it does not admit a finite norm. Time evolution for a quasi-stationary state is given by a semigroup with parameter $t \geq 0$. Due to the Stone-von Neumann theorem this description is not possible under "Hilbert space boundary conditions".

In fact, nature may require boundary conditions other than the Hilbert space axioms. We suggest that dynamical equations like equation (1) which has time symmetric solutions in Hilbert space, will also admit time asymmetric solutions under different time asymmetric boundary conditions. Time asymmetry in quantum mechanics means time asymmetric boundary conditions for time symmetric dynamical equation (1).

A well known example of a time asymmetric boundary condition for time symmetric dynamical equations is the radiation arrow of time. Maxwell's equations (dynamical differential

\footnotetext{
${ }^{2}$ Or another type of paradigm.
} 
equations) are symmetric in time. A boundary condition excludes the strictly incoming fields and selects only the retarded fields of the other sources in the region:

$$
A^{\mu}(x)=A_{\text {ret }}^{\mu}(x)+A_{\text {in }}^{\mu}(x)=A_{\text {ret }}^{\mu}(x) .
$$

The condition $A_{\mathrm{in}}^{\mu}(x)=0$ is the Sommerfeld radiation condition. The boundary condition chooses of the two solutions for the Maxwell equations

$$
A_{\mp}^{\mu}(\vec{x}, t)=\int \delta\left(t^{\prime}-\left(t \mp \frac{\left|\vec{x}-\vec{x}^{\prime}\right|}{c}\right)\right) \frac{j^{\mu}\left(\vec{x}^{\prime}, t^{\prime}\right)}{\left|\vec{x}-\vec{x}^{\prime}\right|} d^{3} x^{\prime} d t^{\prime}
$$

only the retarded solution

$$
A_{\mathrm{ret}}^{\mu}(\vec{x}, t) \equiv A_{-}^{\mu}(\vec{x}, t)=\int \frac{j^{\mu}\left(\vec{x}^{\prime}, t-\frac{\left|\vec{x}-\vec{x}^{\prime}\right|}{c}\right)}{\left|\vec{x}-\vec{x}^{\prime}\right|} d^{3} x^{\prime} .
$$

However, in standard quantum mechanics one mostly does not use time-asymmetric boundary conditions, but instead uses the Hilbert space axiom for the solutions of the Schrödinger (or Heisenberg) equation. To obtain time asymmetric boundary conditions in quantum theory, we need to revise some basic ideas about the boundary conditions for states. The same should be the case for observables in the Heisenberg representation. These ideas [2, 3, 4, 5, 6, 7] are the subject of the next section.

\section{The notions of state and observable revisited}

Let us consider a scattering experiment in the non-relativistic case. In a scattering experiment, a state is "created" or prepared by a preparation apparatus. This state interacts with a center of forces and as the result of this interaction a new state emerges which can be observed in the distant future by a registration apparatus. In this context [2], we have to define the notions of state and observable in the following way:

States are described by a density operator $\rho$ or by a state vector $\phi$.

Observables are described by selfadjoint operators $A=A^{\dagger}$, e.g., by a projection operator $\Lambda=\Lambda^{2}$; if an observable is given by $\Lambda=|\psi\rangle\langle\psi|$ then $\psi$ (and every $e^{i \alpha} \psi, \alpha$ real) will be called an observable vector with the property $\Lambda$.

States are prepared by a preparation apparatus, like an accelerator. Thus, in a scattering experiment a state is identified with what is usually called an incoming state. On the other hand, an observable is registered by a registration apparatus, e.g., a detector. Detected outgoing states are really observables $|\psi(t)\rangle\langle\psi(t)|$ and observables obey the Heisenberg equation of motion. The reason for this is that the experimental quantities are the Born probabilities to measure an observable $\Lambda$ in the state $\rho$ described by $\operatorname{Tr}(\Lambda \rho)$. They are calculated in theory as the Born probabilities and they are measured as the ratio of large numbers of detector counts $N(t) / N$. In the Schrödinger picture they are given by:

$$
\frac{N(t)}{N} \approx \mathcal{P}_{\rho(t)}(\Lambda) \equiv \operatorname{Tr}\left(\Lambda_{0} \rho(t)\right)=\left|\left\langle\psi^{-} \mid \phi^{+}(t)\right\rangle\right|^{2} .
$$

The signs + and - are chosen in agreement with signs as they will appear in the LippmannSchwinger equations in Section 3.1. In particular, this means that $\phi^{+}=\Omega_{\mathrm{IN}} \phi^{\text {in }}$ and $\psi^{-}=$ $\Omega_{\text {OUT }} \psi^{\text {out }}$, where "in" and "out" means incoming and outgoing respectively.

In the Heisenberg picture the Born probabilities are given by:

$$
\frac{N(t)}{N} \approx \mathcal{P}_{\rho}(\Lambda(t)) \equiv \operatorname{Tr}\left(\Lambda(t) \rho_{0}\right)=\left|\left\langle\psi^{-}(t) \mid \phi^{+}\right\rangle\right|^{2}
$$


with time dependent observable $\Lambda(t)$ or $|\psi(t)\rangle\langle\psi(t)|$. The sign $\approx$ indicates that these equalities are the comparison between the experimental quantity, the counting rates $N(t) / N$, and the calculated, theoretical Born probabilities. The equivalence between the Schrödinger and Heisenberg pictures is given by the following mathematical identity:

$$
\operatorname{Tr}\left(\left|\psi^{-}\right\rangle\left\langle\psi^{-} \mid \phi^{+}(t)\right\rangle\left\langle\phi^{+}(t)\right|\right)=\left|\left\langle\psi^{-} \mid \phi^{+}(t)\right\rangle\right|^{2}=\left|\left\langle\psi^{-}(t) \mid \phi^{+}\right\rangle\right|^{2}=\operatorname{Tr}(|\psi(t)\rangle\langle\psi(t) \mid \phi\rangle\langle\phi|) .
$$

We ask the following question: is there any evidence that the time $t$ may take any value? This means, is there any evidence that $U(t)=e^{i H t / \hbar}$ in the Heisenberg picture and $e^{-i H t / \hbar}$ in the Schrödinger picture makes sense for any value of $t$ in $(-\infty, \infty)$ ? It seems obvious that, in analogy to the radiation arrow of time, a state $\phi$ must be prepared at a time $t_{0}$ before the observable $|\psi(t)\rangle\langle\psi(t)|$ can be measured in it. This property is a form of causality principle, e.g., the detector cannot count the decay products $|\psi(t)\rangle\langle\psi(t)|$ before the decaying state $|\phi\rangle\langle\phi|$ has been prepared.

In this sense, we have a Quantum Mechanical Arrow of Time. The Born probability to measure the observable $|\psi(t)\rangle\langle\psi(t)|$ in the state $\phi$,

$$
\mathcal{P}_{\phi}(\psi(t))=|\langle\psi(t) \mid \phi\rangle|^{2}=\left|\left\langle e^{i H t / \hbar} \psi \mid \phi\right\rangle\right|^{2}=\left|\left\langle\psi \mid e^{-i H t / \hbar} \phi\right\rangle\right|^{2}=|\langle\psi \mid \phi(t)\rangle|^{2}=\mathcal{P}_{\phi(t)}(\psi),
$$

exists (experimentally) only for $t \geq t_{0}(=0)$ where $t_{0}$ is the time at which the state has been prepared and the observable can be detected or "registered", i.e., $t_{0}$ is the preparation time of the state $\phi$.

In contrast, the Hilbert space axiom (of conventional QM) predicts $|\langle\psi(t) \mid \phi\rangle|^{2}$ for all $-\infty<t<+\infty$, by the Stone theorem.

In order to obey the causality principle we have to find a theory for which the solutions of the Schrödinger equation $\phi(t)$ evolve by a semigroup $\mathcal{U}_{-}(t)=e^{-i H_{-} t / \hbar}, t_{0}=0 \leq t<\infty$, or for which the solutions of the Heisenberg equation, $\psi(t)$, evolve by a semigroup $\mathcal{U}_{+}(t)=e^{+i H_{+} t / \hbar}$, $0 \leq t<\infty$. By $H_{-}$and $H_{+}$we denote the generators of the semigroups $\mathcal{U}_{-}(t), 0 \leq t<\infty$, and $\mathcal{U}_{+}(t), 0 \leq t<\infty$, respectively.

From a mathematical point of view $\mathcal{U}_{ \pm}^{\times}$are the extensions of the Schrödinger evolution operator beyond Hilbert space to the duals of two rigged Hilbert spaces. This extensions exist only for $0 \leq t<\infty$. Analogously, $H_{ \pm}^{\times}$are the respective extensions of the Hilbert space Hamiltonian $H$. These extensions will be introduced in Section 3.2.

Then, the Born probability for the observable $|\psi\rangle\langle\psi|$ in the state $\phi$ is predicted in this time asymmetric theory for $t \geq t_{0}=0$ only

$$
\mathcal{P}_{\phi}(\psi(t))=|\langle\psi \mid \phi(t)\rangle|^{2}=\left|\left\langle\psi \mid e^{-i H t / \hbar} \phi\right\rangle\right|^{2}=\left|\left\langle e^{i H t / \hbar} \psi \mid \phi\right\rangle\right|^{2}=|\langle\psi(t) \mid \phi\rangle|^{2}, \quad t \geq t_{0}=0 .
$$

Here $t_{0}=0$ represents the preparation time of the state $\phi$. The question is then: can one observe this time $t_{0}$ and how can one observe it? As is common for quantum physical values one expects that $t_{0}$ is measured by an ensemble of values $\left\{t_{0}^{(n)}\right\}$ [8]. Two examples are discussed in [2]. These examples may show evidence that $i$ ) states can be prepared during a finite time; ii) events can be detected individually.

In summary, the detector cannot count the decay products of a state before this state has been prepared. This conclusion is the principle of causality. It means that we have a Quantum Mechanical Arrow of Time (QMAT) which can be formulated as follows:

The Born probability to measure the observable $|\psi(t)\rangle\langle\psi(t)|$ in the state $\phi$ given by $\mathcal{P}_{\phi}(\psi(t))$ in (2) exists experimentally only for $t \geq t_{0}(=0)$, where $t_{0}$ is the preparation time of the state $\phi$.

The task is to find a theory for which the solutions of the Schrödinger equation, $\phi(t)$, evolve by a semigroup $\mathcal{U}_{-}(t)=e^{-i H_{-} t / \hbar}, 0 \leq t<\infty$ or for which the solutions of the Heisenberg equation, $\psi(t)$, evolve by the semigroup $\mathcal{U}_{+}(t)=e^{+i H_{+} t / \hbar}, 0 \leq t<\infty$. Here $H_{ \pm}$are the generators of the semigroups $\mathcal{U}_{ \pm}(t)$, respectively. This task will be discussed in the next section. 


\section{From the mathematics for Dirac kets to separate representation for states and for observables}

In the previous section we have established the need for time asymmetry in quantum mechanics. A preliminary step towards this formulation of Time Asymmetric Quantum Mechanics (TAQM) is the Mathematical Theory of Dirac kets using Gel'fand Triplets based on the Schwartz space Gel'fand triplet:

$$
\Phi \subset \mathcal{H} \subset \Phi^{\times} .
$$

Here $\Phi$ is a dense subspace of $\mathcal{H}$ endowed with a finer topology than the topology inherited from $\mathcal{H}$, and $\Phi^{\times}$is the (topological) dual of $\Phi$ formed by the space of continuous antilinear ${ }^{3}$ functionals on $\Phi$.

The most popular example of a RHS is given by the Schwartz space triplet with $\Phi \doteqdot \mathcal{S}$, then, $\mathcal{H}$ is $L^{2}(\mathbb{R})$, the space of complex valued Lebesgue square integrable functions. The dual space of $\mathcal{S}$ is the space of the (antilinear) tempered distributions $\Phi^{\times} \doteqdot \mathcal{S}^{\times}$. This gives the Dirac ket the mathematical meaning of an antilinear continuous functional in the Schwartz space, $|E\rangle \in \Phi^{\times}$.

The use of Gel'fand triples [9, 10] to implement the Dirac formulation [11] of quantum mechanics has been suggested copiously $[3,12,13,14]$. Dirac kets are well defined as functionals in $\Phi^{\times}$. For certain simple systems (like the free particle or the harmonic oscillator), the Dirac formulation can be implemented by using the Schwartz space. The use of the Schwartz space can be applied to the energy representation $[13,15]$, in which case the wave functions are functions of the energy. In this case, the Schwartz space is used to implement both the space of states $\{\phi\}$ and the space of observables $\{\psi\}$, so that $\{\phi\} \equiv\{\psi\} \equiv \Phi$. In applications to quantum mechanics the values of energy are bounded from below; the Schwartz space that is used as a realization of $\Phi$ is then no longer $\mathcal{S}$ but instead the space of Schwartz functions that vanish on the negative semiaxis: $\Phi \doteqdot \mathcal{S}_{\mathbb{R}^{+}}$. For any pair of functions $\psi, \phi \in \Phi$ with $\psi(E), \phi(E) \in \mathcal{S}_{\mathbb{R}^{+}}$, we have

$$
(\psi, \phi)=\int_{0}^{\infty}\langle\psi \mid E\rangle\langle E \mid \phi\rangle d E, \quad \psi^{*}(E)=\langle\psi \mid E\rangle, \quad \phi(E)=\langle E \mid \phi\rangle \in \mathcal{S}_{\mathbb{R}^{+}}
$$

The kets $|E\rangle$ are generalized eigenvectors of the Hamiltonian with eigenvalue $E$ in the continuous spectrum of $H,\langle\psi|H| E\rangle=E\langle\psi \mid E\rangle$ for all $\psi \in \Phi$. In most cases, the energy (or $H$ ) does not form a complete system of commuting observables (csco), so that some additional observables should be added to $H$ in order to obtain a csco. These are often the angular momentum and the third component of the angular momentum, spin, etc. This is the reason why we choose for the generalized eigevectors of $H$ the kets $\left|E, j, j_{3}, \eta\right\rangle$, which are generalized eigenvectors of $H$ and of angular momentum operators $J^{2}$ and $J_{3}: H^{\times}\left|E, j, j_{3}, \eta\right\rangle=E\left|E, j, j_{3}, \eta\right\rangle$, and of other observables called $\eta$ here. Here $H^{\times}$is the extension of $H$ to $\Phi^{\times}$. In these examples any vector in $\Phi$ admits the following basis vector expansion in terms of the generalized eigenvectors of $H$ :

$$
\phi=\sum_{j, j_{3}, \eta} \int_{0}^{\infty}\left|E, j, j_{3}, \eta\right\rangle\left\langle E, j, j_{3}, \eta \mid \phi\right\rangle d E .
$$

In terms of the wave functions, (5) implies:

$$
\left\langle E, j, j_{3}, \eta|H| \phi\right\rangle=E\left\langle E, j, j_{3}, \eta \mid \phi\right\rangle=E \phi_{j, j_{3}, \eta}(E),
$$

\footnotetext{
${ }^{3}$ The action $F \in \Phi^{\times}$on a certain $\phi \in \Phi$ is a complex number denoted by $\langle\phi \mid F\rangle$. If $f \in \mathcal{H}$, it defines an element of $\Phi^{\times}, F_{f}$, defined by $\left\langle\phi \mid F_{f}\right\rangle=\langle\phi \mid f\rangle$, where $\langle\phi \mid f\rangle$ is the ordinary scalar product on $\mathcal{H}$. This notation is convenient not only because the action of $F$ on $\phi$ extends the scalar product, but also because the scalar product is antilinear to the left.
} 
which means that, in the energy representation, the Hamiltonian $H$ is represented by the multiplication operator, as it should. In the energy representation, the Gel'fand triplet $\Phi \subset \mathcal{H} \subset \Phi^{\times}$ is represented by $\mathcal{S}_{\mathbb{R}^{+}} \subset L^{2}\left(\mathbb{R}^{+}\right) \subset\left(\mathcal{S}_{\mathbb{R}^{+}}\right)^{\times}$.

In the energy representation, both states $\phi$ and observables $|\psi\rangle\langle\psi|$ are given by respective wave functions $\phi(E)$ and $\psi(E)$ that belong to the Schwartz space $\mathcal{S}_{\mathbb{R}^{+}}$. Due to a mathematical theorem [13, p. 82] the time evolution in either Schrödinger and Heisenberg picture extends also to the Schwartz space triplet (3) for all values of $t:-\infty<t<\infty$.

However, there is no reason that the set of observables $|\psi\rangle\langle\psi|, A$, and the set of states $\phi, \rho$, should be described by the same space; i.e., that $\{\phi\}=\{\psi\}$, and that these should be given by the Schwartz space.

As mentioned above, in scattering experiments one distinguishes between the in-states $\phi^{+}$and out-states $\psi^{-}$; but one is not much concerned with the mathematical properties of the spaces of in-states $\left\{\phi^{+}\right\}$and of out-states $\left\{\psi^{-}\right\}$. The in-states are prepared by a preparation apparatus (e.g., an accelerator) and therefore obey the Schrödinger equation. The out-entities are not really states because they are the entities registered by a detector; therefore the $\psi^{-}$represent observables, physically defined by the registration apparatus (e.g., a detector). Therefore the $\psi^{-}$ should obey the Heisenberg equation of motion, i.e.,

$$
\begin{aligned}
& i \hbar \frac{\partial}{\partial t}\left|\psi^{-}(t)\right\rangle\left\langle\psi^{-}(t)\right|=-\left[H,\left|\psi^{-}(t)\right\rangle\left\langle\psi^{-}(t)\right|\right], \\
& i \hbar \frac{\partial}{\partial t} \psi^{-}=-H \psi^{-} .
\end{aligned}
$$

The time evolution would then be given not by the Schrödiger equation (1), but it would be the time evolution of observables $A$, i.e., it would be given by

$$
A(t)=\mathrm{e}^{i H t / \hbar} A \mathrm{e}^{-i H t / \hbar} \quad \text { or } \quad \psi(t)=\mathrm{e}^{i H t / \hbar} \psi .
$$

Thus we would expect that one needs not one RHS (3), but a pair of RHS's

$$
\left\{\psi^{-}\right\} \equiv \Phi_{+} \subset \mathcal{H} \subset \Phi_{+}^{\times} \quad \text { and } \quad\left\{\phi^{+}\right\} \equiv \Phi_{-} \subset \mathcal{H} \subset \Phi_{-}^{\times} .
$$

One RHS is for the observables $\left\{\psi^{-}\right\}$fulfilling the Heisenberg equation of motion (6b), and the other RHS is for the states $\left\{\phi^{+}\right\}$fulfilling the Schrödinger equation (1). This idea is supported by the phenomenological theories of scattering and decay ${ }^{4}$.

\subsection{From the phenomenological theories of scattering and decay to a pair of Hardy spaces}

To conjecture mathematical properties of the pair of RHS's (7) we use as point of departure the in- and out- plane wave kets $\left|E^{+}\right\rangle$and $\left|E^{-}\right\rangle$which fulfill the Lippmann-Schwinger equation ${ }^{5}$ $[20,21,22,23]$ :

$$
\left|E^{ \pm}\right\rangle=|E\rangle+\frac{1}{E-H \pm i \epsilon} V|E\rangle=\Omega^{ \pm}|E\rangle, \quad \epsilon \rightarrow+0
$$

The vectors $\left|E^{+}\right\rangle, E \in \mathbb{R}^{+}=[0, \infty)$, are taken as a basis systems for the Dirac basis vector expansions of in-state vectors $\phi^{+}$as ${ }^{6}$

$$
\phi^{+}=\sum_{j, j_{3}, \eta} \int_{0}^{\infty}\left|E, j, j_{3}, \eta^{+}\right\rangle\left\langle{ }^{+} E, j, j_{3}, \eta \mid \phi^{+}\right\rangle d E=\int_{0}^{\infty}\left|E^{+}\right\rangle\left\langle{ }^{+} E \mid \phi^{+}\right\rangle d E
$$

\footnotetext{
${ }^{4}$ The notation $\phi^{+} \in \Phi_{-}$and $\psi^{-} \in \Phi_{+}$may appear awkward, it has its origin in the difference of notation in the mathematics literature for Hardy spaces [16, 17, 18, 19] $\Phi \mp$, and in the physics literature for in and out states $\phi^{ \pm}\left(\phi^{ \pm} \in \Phi_{\mp}\right)$ of scattering theory $[2,20,21,22]$.

${ }^{5}$ Here $\Omega^{-}=\Omega_{\text {OUT }}$ and $\Omega^{+}=\Omega_{\text {IN }}$.

${ }^{6}$ We assume the absence of bound states for simplicity.
} 
and vectors $\left|E^{-}\right\rangle$are taken as basis vectors for the Dirac expansion of the out-vectors for the observables $|\psi\rangle\langle\psi|$ :

$$
\psi^{-}=\sum_{j, j_{3}, \eta} \int_{0}^{\infty}\left|E, j, j_{3}, \eta^{-}\right\rangle\left\langle^{-} E, j, j_{3}, \eta \mid \psi^{-}\right\rangle d E=\int_{0}^{\infty}\left|E^{-}\right\rangle\left\langle^{-} E \mid \psi^{-}\right\rangle d E .
$$

The expansions (9), (10) are statement of the nuclear spectral theorem for the pair of the RHS's (7) [9, 10, 13, 15].

As was stated previously, $j, j_{3}, \eta$ denote the additional quantum numbers. Here $j, j_{3}$ have been chosen to correspond to the angular momentum and $\eta$ to particle species labels, e.g., channel quantum numbers etc. The Dirac basis vector expansions (9) and (10) use two different kinds of kets: $\left|E^{\mp}\right\rangle=\left|E j j_{3} \eta^{\mp}\right\rangle \in \Phi_{ \pm}^{\times}$, as suggested by (8), the Lippmann-Schwinger out-plane waves $\left|E^{-}\right\rangle$and in-plane waves $\left|E^{+}\right\rangle$, respectively. These kets should belong to the duals in certain Gel'fand triplets (7).

The properties of the Ge'lfand triplets (7) are defined by the mathematical properties of the set of the energy wave functions in the same way as the Schwartz space $\Phi$ of (3) is defined by the set of energy wave functions $\mathcal{S}_{\mathbb{R}^{+}}=\{\phi(E)\}$ in (4). The properties of the energy wave functions $\phi^{+}(E)=\left\langle{ }^{+} E \mid \phi^{+}\right\rangle$in (9) and of $\psi^{-}(E)=\left\langle{ }^{-} E \mid \psi^{-}\right\rangle$in (10) have been obtained from the phenomenology of resonance and decay phenomena [24]. From these properties the functions

$$
\phi^{+}(E) \equiv\left\langle{ }^{+} E \mid \phi^{+}\right\rangle=\left\langle{ }^{+} E j j_{3} \eta \mid \phi^{+}\right\rangle=\left\langle\phi^{+} \mid E j j_{3} \eta^{+}\right\rangle^{*}
$$

were identified as the boundary value on the positive semiaxis $\mathbb{R}^{+}$of an Hardy function in the lower complex energy half-plane [25]. It is convenient to define this analytic function on the lower half plane which corresponds to the second sheet of the Riemann surface that supports the values of the $S$-matrix $S_{j}(z)$.

Similarly, the energy wave function of the observable $\left|\psi^{-}\right\rangle\left\langle\psi^{-}\right|$

$$
\psi^{-}(E) \equiv\left\langle^{-} E \mid \psi^{-}\right\rangle=\left\langle{ }^{-} E j j_{3} \eta \mid \psi^{-}\right\rangle
$$

can be extended into an Hardy function in the upper complex energy half-plane [25] on the second sheet of the Riemann surface corresponding to the $S$-matrix. Then $\left\langle\psi^{-} \mid E^{-}\right\rangle{ }^{+} E\left|\phi^{+}\right\rangle$ in the $S$-matrix element (13) below can be continued into the lower complex energy semi-plane (2-nd sheet of the $S$-matrix).

The situation can be summarized in the following diagram:

$\begin{array}{llll}\text { Two sets of Hardy functions } & \begin{array}{l}\text { for the two } \\ \text { sets of vectors }\end{array} & \begin{array}{l}\text { from two sets } \\ \text { of L-Sch. kets }\end{array} & \begin{array}{l}\text { leading to two } \\ \text { Hardy spaces }\end{array}\end{array}$

$$
\begin{array}{llll}
\left\{\phi^{+}(z)=\left\langle{ }^{+} z \mid \phi^{+}\right\rangle\right\} & \left\{\phi^{+}\right\}=\text {states } & \left|E^{+}\right\rangle=\left|E, j, j_{3}, \eta^{+}\right\rangle & \Phi_{-} \\
\left\{\psi^{-}(z)=\left\langle{ }^{-} z \mid \psi^{-}\right\rangle=\left\langle\psi^{-} \mid z^{-}\right\rangle^{*}\right\} & \left\{\psi^{-}\right\}=\text {observables } & \left|E^{-}\right\rangle=\left|E, j, j_{3}, \eta^{-}\right\rangle & \Phi_{+}
\end{array}
$$

From the analyticity property follows that $\left\langle\psi^{-} \mid E^{-}\right\rangle\left\langle E^{+} \mid \psi^{+}\right\rangle S_{j}(E)$ can be analytically continued into the lower complex semi-plane (second sheet), except for singularities of $S_{j}(E)$.

This means that the contour of integration of the $S$-matrix element

$$
\left(\psi^{-}, \phi^{+}\right)=\left(\psi^{\text {out }}, S \phi^{\text {in }}\right)=\sum_{j} \int_{0}^{\infty} d E \sum_{j_{3}} \sum_{\eta, \eta^{\prime}}\left\langle\psi^{-} \mid E, j, j_{3}, \eta^{-}\right\rangle S_{j}^{\eta^{\prime} \eta}(E)\left\langle{ }^{+} E, j, j_{3}, \eta \mid \phi^{+}\right\rangle .
$$

can be deformed into the lower complex energy plane, second sheet of the $S$-matrix element $S_{j}(E)$. 


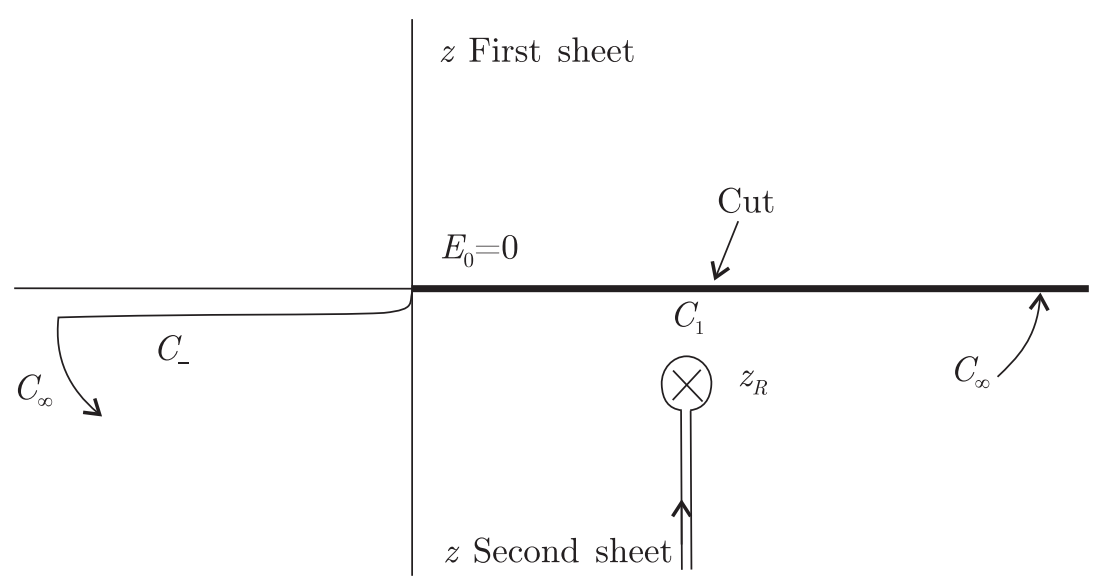

Figure 1. The second sheet of the the complex energy Riemann surface for the $S$-matrix with one resonance pole.

Consider the simplest case that there is one $S$-matrix pole at $z_{R}=E_{R}-i \Gamma / 2$ on the second sheet as shown in Fig. 1. The program to determine the property of the spaces $\Phi_{-}$(states) and $\Phi_{+}$(observables) is the following:

Start with the integral (13) along the cut. In simplified notation the $S$-matrix element (13) is given by

$$
\left(\psi^{-}, \phi^{+}\right)=\int_{0}^{\infty} d E\left\langle\psi^{-} \mid E^{-}\right\rangle S_{j}(E)\left\langle^{+} E \mid \phi^{+}\right\rangle .
$$

The contour of integration is now deformed from the positive real axis first sheet through the cut into the lower complex energy plane in the second sheet, where the resonance pole of the $S$-matrix is located at $E=z_{R}$. Then one obtains the integrals along $\mathcal{C}_{-}$, along the semicircle $\mathcal{C}_{\infty}$ and the integral $\mathcal{C}_{1}$ around the pole at $z_{R}$. This is depicted in Fig. 1 . The integral along $\mathcal{C}_{\infty}$ vanishes and the integral along $\mathcal{C}_{-}$gives some non-resonant background, whereas we wish to determine the needed properties of the energy wave functions (11), (12) from the resonance and decay properties associated to the $S$-matrix pole at $z_{R}$.

The task is: conjecture the mathematical property of $\left\langle{ }^{-} E \mid \psi^{-}\right\rangle,\left\langle{ }^{+} E \mid \phi^{+}\right\rangle$such that a scattering resonance and a decaying state is the same physical entity derived from the $S$-matrix pole at $z_{R}$ on the second sheet.

\subsection{Conjecturing the Hardy space axiom}

We want to find the mathematical definition of the spaces $\left\{\phi^{+}\right\}$and $\left\{\psi^{-}\right\}$. For this purpose we start with the $S$-matrix pole definition of a resonance at $z_{R}=E_{R}-i \Gamma / 2$ on the second sheet of the $j$-th partial $S$-matrix element $S_{j}(E)$.

We obtain (using the Cauchy theorem) from the pole term of $S_{j}^{\text {P.T. }}(E)=2 i a_{j}^{\mathrm{BW}}(E)$ around $z_{R}$ a Breit-Wigner resonance amplitude $a_{j}^{\mathrm{BW}}(E)$

$$
a_{j}^{\mathrm{BW}}(E)=\frac{R}{E-E_{R}-i \Gamma / 2}(15 \mathrm{a}) \Leftrightarrow \quad\left|z_{R}, j, j_{3}, \eta\right\rangle \sqrt{2 \pi \Gamma}=\int_{-\infty}^{+\infty} d E\left|E, j, j_{3}, \eta^{-}\right\rangle \frac{i \sqrt{\frac{\Gamma}{2 \pi}}}{E-z_{R}}
$$

For the Gamow ket one can show that it is an eigenket of $H$ with a discrete complex eigenvalue (as Gamow wanted):

$$
H^{\times}\left|E_{R}-i \Gamma / 2, j, j_{3}, \eta^{-}\right\rangle=\left(E_{R}-i \Gamma /\right)\left|E_{R}-i \Gamma / 2, j, j_{3}, \eta^{-}\right\rangle .
$$


Further one can show that the Gamow ket has the property:

$$
\begin{aligned}
& \left\langle\mathrm{e}^{i H t / \hbar} \psi_{\eta}^{-} \mid E_{R}-i \Gamma / 2, j, j_{3}, \eta^{-}\right\rangle=\left\langle\psi_{\eta}^{-}\left|\mathrm{e}^{-i H^{\times} t / \hbar}\right| E_{R}-i \Gamma / 2, j, j_{3}, \eta^{-}\right\rangle \\
& \quad=\mathrm{e}^{-i E_{R} t / \hbar} \mathrm{e}^{-(\Gamma / 2) t / \hbar}\left\langle\psi_{\eta}^{-} \mid E_{R}-i \Gamma / 2, j, j_{3}, \eta^{-}\right\rangle \quad \text { but for } t \geq 0\left(=t_{0}\right) \text { only. }
\end{aligned}
$$

This proves that the Breit-Wigner resonance observed by the Breit-Wigner cross section

$$
\sigma_{j}^{R}(E)=\frac{4 \pi}{p^{2}}(2 j+1) \frac{(\Gamma / 2)^{2}}{\left(E-E_{R}\right)^{2}+(\Gamma / 2)^{2}}
$$

(i.e., by the lineshape (17) as a function of $E$ ), is the same physical object as the exponentially decaying state $(16 \mathrm{~b})$, observed by the exponential time evolution:

$$
\left|\left\langle\mathrm{e}^{i H t / \hbar} \psi_{\eta}^{-} \mid E_{R}-i \Gamma / 2, j, j_{3}, \eta^{-}\right\rangle\right|^{2}=\mathrm{e}^{-\Gamma t / \hbar}\left|\left\langle\psi_{\eta}^{-} \mid E_{R}-i \Gamma / 2, j, j_{3}, \eta^{-}\right\rangle\right|^{2} .
$$

This means that the ket (15b) with Breit-Wigner resonance distribution is a Gamow ket (16a) with exponential time evolution (18):

Breit-Wigner resonance of width $\Gamma \equiv$ a decaying state with lifetime $\tau=\hbar / \Gamma$.

Therewith we have a theory that unifies the concept of a Breit-Wigner resonance (15a), (17) and the concept of an exponentially decaying Gamow states (15b), (18).

In order to separate the pole term in (15a) from the $S$-matrix in (14) and associate to it a ket $\left|z_{R}=\left(E_{R}-i \Gamma / 2\right), j, j_{3}, \eta\right\rangle$ in (15b) with a Breit-Wigner energy distribution extending over $-\infty<E<+\infty$ (and not over the spectrum $E_{0}=0 \leq E<\infty$ of the Hilbert space operator $H$ in (9) and (10)), and in order to derive (16a), (16b) and (18) as generalized eigenvector equations, new mathematical conditions must be met by the energy wave functions $\left\langle{ }^{-} E \mid \psi^{-}\right\rangle$and ${ }^{+} E\left|\phi^{+}\right\rangle$ of (11), (12) in addition to the usual analyticity properties in the upper and lower complex semi-plane, and in addition to their Schwartz space property ${ }^{7}$ on the positive real axis $\mathbb{R}$.

From these mathematical conditions the energy wave functions were identified [25] as Hardy functions of the complex energy semi-planes and this hypothesis was discussed subsequently in several publications [16, 17, 26, 27].

Specifically, the energy wave functions of a state must be smooth Hardy functions on the lower complex plane $\mathcal{C}_{-}$. In our context this lower complex plane is taken as the second sheet of the Riemann surface of the $S$-matrix:

$$
\phi^{+}(E)=\left.\left\langle{ }^{+} E \mid \phi^{+}\right\rangle \in\left(\mathcal{H}_{-}^{2} \cap \mathcal{S}\right)\right|_{\mathbb{R}_{+}} \equiv \text { Hardy functions on the lower semi-plane } \mathcal{C}_{-} .
$$

The energy wave functions of an observable are smooth Hardy functions analytic on the upper semi-plane $\mathcal{C}_{+}$(so that their complex conjugates are analytic on the lower semi-plane):

$$
\psi^{-}(E)=\left.\left\langle{ }^{-} E \mid \psi^{-}\right\rangle \in\left(\mathcal{H}_{+}^{2} \cap \mathcal{S}\right)\right|_{\mathbb{R}_{+}} \equiv \text { Hardy functions on the upper semi-plane } \mathcal{C}_{+} .
$$

Therewith we have inferred a new axiom of a causal quantum theory which provides a mathematical description that unifies resonance and decay phenomena:

\section{Hardy space axiom}

The set of prepared (in-) states defined by the preparation apparatus (e.g. accelerator) is described by the triplet

$$
\left\{\phi^{+}\right\} \equiv \Phi_{-} \subset \mathcal{H} \subset \Phi_{-}^{\times} .
$$

\footnotetext{
${ }^{7}$ Infinitely differentiable and rapidly decreasing.
} 
The set of (out-) observables defined by the registration apparatus (e.g. detector) is described by the triplet

$$
\left\{\psi^{-}\right\} \equiv \Phi_{+} \subset \mathcal{H} \subset \Phi_{+}^{\times}
$$

The spaces $\Phi_{ \pm}$are the Hardy spaces on the semi-planes $\mathcal{C}_{ \pm}$(second sheet of the analytic $S$ matrix), and $\Phi_{ \pm}^{\times}$are their respective duals ${ }^{8}$.

The Lippmann-Schwinger kets $\left|E, j, j_{3}, \eta^{ \pm}\right\rangle$the Gamow kets $\left|R_{R}-i \Gamma / 2, j, j_{3}, \eta^{-}\right\rangle$and $\left|z, j, j_{3}, \eta^{ \pm}\right\rangle$are mathematically well defined as functionals on the space $\Phi_{\mp}$.

To distinguish in the mathematical description between states $\left\{\phi^{+}\right\} \equiv \Phi_{-}$and the observables $\left\{\psi^{-}\right\} \equiv \Phi_{+}$is quite natural, since experimentalists distinguish between preparation apparatus (e.g., accelerator) for states and registration apparatus (detector) for observables.

Having discovered the Hardy space axiom for the quantum theory of resonances and decaying states one can derive mathematical consequences of this axiom.

In the same way as the unitary group evolution followed by the Stone-von Neumann theorem from the Hilbert space axiom, the time evolution for the Hardy space axiom follows from a mathematical theorem for Hardy spaces, the Paley-Wiener theorem (1934) [18]:

The solutions of the dynamical equations, the Schrödinger equation for the state $\phi^{+}(t)$ and the Heisenberg equation for the observable $\psi^{-}(t)$ are given by the semigroup

(Heisenberg equation):

of observables in space $\Phi_{+}$

$$
\begin{gathered}
\psi^{-}(t)=e^{i H t / \hbar} \psi^{-} \\
t_{0}=0 \leq t<\infty
\end{gathered}
$$

(Schrödinger equation):

for states in the space $\Phi_{-}$

$$
\begin{gathered}
\phi^{+}(t)=e^{-i H t / \hbar} \phi^{+} \\
t_{0}=0 \leq t<\infty .
\end{gathered}
$$

Therefore the probability for the time evolved observable $\psi^{-}(t)$ in the state $\phi^{+}$can now be predicted as the Born probability:

$$
\begin{aligned}
\mathcal{P}_{\phi^{+}}\left(\psi^{-}(t)\right) & =\left|\left\langle\psi^{-}(t) \mid \phi^{+}\right\rangle\right|^{2}=\left|\left\langle e^{i H t / \hbar} \psi^{-} \mid \phi^{+}\right\rangle\right|^{2} \\
& =\left|\left\langle\psi^{-} \mid e^{-i H_{+} t / \hbar} \phi^{+}\right\rangle\right|^{2} \quad \text { for } t \geq t_{0}=0 \text { only. }
\end{aligned}
$$

This is in agreement with experimental data for the detector counts because

$$
\mathcal{P}_{\phi^{+}}\left(\psi^{-}(t)\right) \sim N(t) / N \text { can be measured only for } t \geq t_{0}=0,
$$

where $t_{0}$ is the time at which the state $\phi^{+}$had been prepared (causality).

\section{Concluding remarks}

In order to derive from the resonance pole of the $S$-matrix $S_{j}(E)$ at $z_{R}=E_{R}-i \Gamma / 2$ the Breit-Wigner resonance amplitude in (15a) and to derive for it the Gamow ket in (15b) with the property (16a), (16b) such that the relation $\tau=\hbar / \Gamma$ is fulfilled, one has to make various mathematical assumptions for the energy wave functions. These mathematical assumptions go beyond the requirement that $\phi^{+}(E)$ and $\psi^{-}(E)$ are the Schwartz functions of the mathematical version of the Dirac formalisms and can be summarized by the new Hardy space axiom (19), (20).

Starting with (19) and (20) as the new axiom for the space of states $\left\{\phi^{+}\right\}=\Phi_{-}$and the space of observables $\left\{\psi^{-}\right\}=\Phi_{+}$one just needs to use the mathematical theorem of Paley and Wiener [18] to obtain a semigroup time evolution (21). For the special case of Gamow kets, one obtains from this new axiom the exponential decay law (18) for the states described by the

\footnotetext{
${ }^{8} \Phi_{ \pm}^{\times}$are spaces of antilinear continuous functionals on $\Phi_{ \pm}$.
} 
Gamow ket. But one obtains (16b), (18) for $t>t_{0}=0$. This is an intuitively plausible result if $t_{0}=0$ is the time at which the Gamow state $\phi^{G}=\sqrt{2 \pi \Gamma}\left|z_{R}, j, j_{3}, \eta\right\rangle$ is prepared.

An interesting question is the interpretation of the time $t_{0}=0$, which could be any finite time in our life or the life of the Universe, but certainly should not be a time before the big bang. Since quantum theory deals with an ensemble of quanta, one would also expect that the "quantum mechanical beginning of time" $t_{0}$ will be observed by an ensemble of times $\left\{t_{0}^{(1)}, t_{0}^{(2)}, \ldots\right\}$. How to interpret this ensemble of times and how to identify these times $t_{0}^{(i)}$ in experiments is an interesting question.

\section{Appendix: Hardy functions on a half plane}

Let us consider the open upper half plane $\mathbb{C}^{+}:=\{z \in \mathbb{C} \mid \operatorname{Im} z>0\}$, where $\mathbb{C}$ is the field of complex numbers. A Hardy function $[13,15,18,28] f(z)$ on the upper half plane is an analytic function on $\mathbb{C}^{+}$such that for any line $y=x+i \alpha, \alpha>0$ and constant (i.e., any parallel to the real axis in $\mathbb{C}^{+}$), one has that

$$
\int_{-\infty}^{\infty}|f(x+i \alpha)|^{2} d x<K<\infty
$$

This $K$ is the same for all values of $\alpha>0$. As a consequence, the function $f(x):=\lim _{\alpha \mapsto 0} f(x+i \alpha)$ is well defined (almost elsewhere) on the real line $\mathbb{R}$ and is square integrable, i.e.,

$$
\int_{-\infty}^{\infty}|f(x)|^{2} d x<K<\infty
$$

where this $K$ is the same we have in (22). Thus, $f(x)$ is the function given by the boundary values on the real axis of $f(z)$ and therefore it is uniquely determined by $f(z)$. Conversely, a theorem due to Titchmarsh [28], based on the Cauchy theorem, shows that we can recover the values of $f(z), z \in \mathbb{C}^{+}$, once we know the function $f(x)$. Thus, a Hardy function on the upper half plane $f(z)$ is uniquely determined by the function given its boundary values on the real $f(x)$ line and therefore, we can identify $f(z)$ and $f(x)$.

Let us call $\mathcal{H}_{+}^{2}$ the set of Hardy functions on the upper half plane. Then, $\mathcal{H}_{+}^{2}$ has the following properties:

i) $\mathcal{H}_{+}^{2}$ is a linear space. Since each $f(x) \in \mathcal{H}_{+}^{2}$ is square integrable, $\mathcal{H}_{+}^{2}$ is a subspace of $L^{2}(\mathbb{R})$.

ii) Assume that $f(x) \in L^{2}(\mathbb{R})$. How can we recognize that $f(x)$ is a function in $\mathcal{H}_{+}^{2}$ ? For that, we have the Paley-Wiener theorem [18], which states that $f(x) \in \mathcal{H}_{+}^{2}$ if and only if it is the Fourier transform of a square integrable function supported on (i.e., that vanishes outside of) the negative semiaxis $\mathbb{R}^{-} \equiv(-\infty, 0]$.

iii) The boundary values on an interval of the real line of a Hardy function on the upper half plane uniquely determine such a function, as it happens for any complex analytic function on the open upper half plane. This is particularly true when this interval is the positive semiaxis $\mathbb{R}^{+} \equiv[0, \infty)$. In this case, we have a formula that recovers all the values of $f(z) \in \mathcal{H}_{+}^{2}$ for $z$ on the closed upper half plane (including the values on the negative semiaxis) from the values of $f(x)$ for $x>0$. Furthermore, it gives a criteria that states when a square integrable function on the positive semiaxis can be continued into a Hardy function in $\mathcal{H}_{+}^{2}$. Both results rely on the properties of the Mellin transform [19].

Hardy functions on the lower half plane $\mathbb{C}^{-} \equiv\{z \in \mathbb{C} \mid \operatorname{Im} z<0\}$ are defined analogously. The space of Hardy functions on the lower half plane are denoted by $\mathcal{H}_{-}^{2}$ and have similar properties than those above stated for functions in $\mathcal{H}_{+}^{2}$, with minor differences. In particular, 
in $i$ ) we have to replace Fourier transforms of function supported on the negative semiaxis by Fourier transforms supported on the positive semiaxis [18].

There are some additional properties that concerns functions in both Hardy spaces $\mathcal{H}_{ \pm}^{2}$ :

1. Both spaces $\mathcal{H}_{ \pm}^{2}$ are closed subspaces of $L^{2}(\mathbb{R})$ and therefore Hilbert spaces with the scalar product of functions in $L^{2}(\mathbb{R})$. Moreover, each function in $\mathcal{H}_{ \pm}^{2}$ is orthogonal to each function in $\mathcal{H}_{\mp}^{2}$. In addition,

$$
L^{2}(\mathbb{R})=\mathcal{H}_{+}^{2} \oplus \mathcal{H}_{-}^{2},
$$

where the symbol $\oplus$ means orthogonal direct sum. This is indeed a consequence of the Paley-Wiener theorem [18].

2. The complex conjugate $f^{*}(x)$ of a function $f(x) \in \mathcal{H}_{ \pm}^{2}$ is in $\mathcal{H}_{\mp}^{2}, f(x) \in \mathcal{H}_{\mp}^{2}$. Moreover, $\left[f\left(z^{*}\right)\right]^{*}=f(z)$, for any $f(z) \in \mathcal{H}_{\mp}^{2}$, where the star denotes complex conjugation.

3. Let us call $\left.\mathcal{H}_{ \pm}^{2}\right|_{\mathbb{R}^{+}}$and $\left.\mathcal{H}_{ \pm}^{2} \cap \mathcal{S}\right|_{\mathbb{R}^{+}}$the spaces of the restrictions to the positive semiaxis $\mathbb{R}^{+}$ of the functions in $\mathcal{H}_{ \pm}^{2}$ or in the intersection $\mathcal{H}_{ \pm}^{2} \cap \mathcal{S}$ of the Hardy spaces with the Schwartz space, respectively. These two spaces are dense in $L^{2}\left(\mathbb{R}^{+}\right)[13]$.

\section{Acknowledgments}

We wish to acknowledge partial financial support by the Spanish Ministry of Science and Innovation through Project MTM2009-10751, the Junta de Castilla y León, through Project GR224 and the US NSF Award no OISE-0421936.

\section{References}

[1] Lee T.D., Particle physics and introduction to field theory, Chapter 13, Contemporary Concepts in Physics, Vol. 1, Harwood Academic Publishers, Chur, 1981.

[2] Bohm A., Harshman N.L., Quantum theory in rigged Hilbert space: irreversibility from causality, in Irreversibility and Causality, Semigroups and Rigged Hilbert Spaces (Goslar, 1996), Lecture Notes in Phys., Vol. 504, Editors A. Bohm, H.D. Doebner and P. Kielanowski, Springer, Berlin, 1998, 181-237, quant-ph/9805063.

[3] Bohm A., Time-asymmetric quantum physics, Phys. Rev. A 60 (1999), 861-876, quant-ph/9902085.

[4] Bohm A., Loewe M., Van de Ven B., Time asymmetric quantum theory. I. Modifying an axiom of quantum physics, Fortschr. Phys. 51 (2003), 551-568, quant-ph/0212130.

[5] Bohm A., Kaldass H., Wickramasekara S., Time asymmetric quantum theory. II. Relativistic resonances from $S$-matrix poles, Fortschr. Phys. 51 (2003), 569-603, hep-th/0212280.

[6] Bohm A., Kaldass H., Wickramasekara S., Time asymmetric quantum theory. III. Decaying states and the causal Poincaré semigroup, Fortschr. Phys. 51 (2003), 604-634, hep-th/0212282.

[7] Bohm A., Sato Y., Relativistic resonances: their masses, widths, lifetimes, superposition, and causal evolution, Phys. Rev. D 71 (2005), 085018, 22 pages, hep-ph/0412106.

[8] Bohm A., Bryant P.W., From Hardy spaces to quantum jumps: a quantum mechanical beginning of time, Internat. J. Theoret. Phys. 50 (2011), 2094-2105, arXiv:1011.4954.

[9] Gel'fand I.M., Vilenkin N.Ya., Generalized functions, Vol. 4, Applications of harmonic analysis, Academic Press, New York, 1964.

[10] Maurin K., Generalized eigenfunction expansions and unitary representations of topological groups, Polish Scientific Publishers, Warszawa, 1968.

[11] Dirac P.A.M., The principles of quantum mechanics, Clarendon Press, Oxford, 1930.

[12] Bohm A., Rigged Hilbert space and the mathematical description of physical systems, Boulder Lecture Notes in Theoretical Physics, Vol. 9A, Gordon and Breach Science Publishers, New York, 1967, 255-317.

[13] Bohm A., Gadella M., Dirac kets, Gamow vectors and Gel'fand triplets, Lecture Notes in Physics, Vol. 348, Springer-Verlag, Berlin, 1989. 
[14] Gadella M., Gómez F., A unified mathematical formalism for the Dirac formulation of quantum mechanics, Found. Phys. 32 (2002), 815-869.

[15] Civitarese O., Gadella M., Physical and mathematical aspects of Gamow states, Phys. Rep. 396 (2004), 41-113.

[16] Baumgärtel H., Time asymmetry in quantum mechanics: a pure mathematical point of view, J. Phys. A: Math. Theor. 41 (2008), 304017, 7 pages.

[17] Baumgärtel H., Resonances of quantum mechanical scattering systems and Lax-Phillips scattering theory, J. Math. Phys. 51 (2010), 113508, 20 pages.

[18] Paley R., Wiener N., Fourier transforms in the complex domain, American Mathematical Society Colloquium Publications, Vol. 19, American Mathematical Society, Providence, RI, 1934.

[19] van Winter C., Complex dynamical variables for multiparticle systems with analytic interactions. I, J. Math. Anal. Appl. 47 (1974), 633-670.

[20] Lippmannn B.A., Schwinger J., Variational principles for scattering processes. I, Phys. Rev. 79 (1950), 469-480.

[21] Gell-Mann M., Goldberger H.L., The formal theory of scattering, Phys. Rev. 91 (1953), 398-408.

[22] Goldberger M.L., Watson K.M., Collision theory, John Wiley \& Sons, Inc., New York - London - Sydney, 1964.

[23] Gadella M., Gómez F., The Lippmann-Schwinger equations in the rigged Hilbert space, J. Phys. A: Math. Gen. 35 (2002), 8505-8511.

[24] Bohm A., Quantum mechanics, 2nd ed., Texts and Monographs in Physics, Springer-Verlag, New York, 1986, Chapter XXI.

[25] Baumgärtel H., A private communication ${ }^{9}$ to A. Bohm, 1978.

[26] Baumgärtel H., Generalized eigenvectors for resonances in the Friedrichs model and their associated Gamow vectors, Rev. Math. Phys. 16 (2006), 61-78, Addendum, Rev. Math. Phys. 19 (2007), 227-229, math-ph/0509062.

[27] Baumgärtel H., Time asymmetry in quantum mechanics: a pure mathematical point of view, J. Phys. A: Math. Theor. 41 (2008), 304017, 7 pages.

[28] Duren P.L., Theory of $H^{p}$ spaces, Pure and Applied Mathematics, Vol. 38, Academic Press, New York London, 1970.

\footnotetext{
${ }^{9}$ H. Baumgartel identified these energy wave functions from their required properties as Hardy functions.
} 